\title{
Localized Tactile Stimulation by Time-Reversal of Flexural Waves: Case Study With a Thin Sheet of Glass
}

\author{
Charles Hudin* Jose Lozada ${ }^{\dagger}$ \\ CEA, LIST, Sensorial and Ambient Interfaces Laboratory, 91191, Gif-sur-Yvette CEDEX, FRANCE \\ Vincent Hayward \\ UPMC Univ. Paris 6, ISIR, Institut des Systemes Intelligents et de Robotique, 75005, Paris, FRANCE
}

\begin{abstract}
This paper addresses the issue of producing localized tactile stimuli on a transparent surface. An approach based on time reversal of acoustic waves is presented and implemented on a thin glass surface actuated by piezoelectric transducers located at the periphery. The physical performance measurement, completed by a user study demonstrates the ability to provide localised and perceivable tactile stimulation.
\end{abstract}

Index Terms: H.5.2 [Information Interfaces and Presentation]: User Interfaces-Haptic I/O

\section{INTRODUCTION}

Tactile feedback is an important component of interaction design research $[13,10]$. Feedback provided through multiple sensory channels can increase task performance, for example in the context of interaction with mobile devices, or more generally, enhance usability $[16,3,17]$. In such applications, stimulating the fingertip through a transparent surface has special importance.

Several approaches have been proposed to elicit tactile sensations from a transparent medium, beyond simply vibrating the front plate or the whole case of a device. When it comes to textures or small tactile variations experienced while sliding a finger on a smooth surface, non-linear acoustic pumping is able modulate friction by vibrating the surface at ultrasonic frequencies to create an air film between the surface and the finger [7, 12, 2, 4, 21]. Electrovibration takes advantage of electrostatic forces to attract the finger toward the surface, resulting in an increase of friction $[14,18,1]$. Of course, the whole surface can be vibrated, provided that the position of the finger be accurately known with a resolution commensurate with the size of the details to be rendered [22], which trades off actuation complexity for sensing complexity.

These techniques do not lend themselves naturally to producing localized stimuli, let alone stimulating multiple fingers independently, unless special precautions are used, such as the use of multiples electrodes for electrovibration [19], reducing an already weak effect, or producing patterns of constructive or destructive wave interferences for acoustic pumping [11], an approach that offers only limited options.

We previously demonstrated that the computational time reversal of acoustic waves was a viable approach to achieve localized tactile stimulation in a transparent medium [8]. With this technique, transducers located at the periphery of a stretched membrane produced flexural waves that propagated to interfere constructively, at a given time and at a given place, to produce localized mechanical deformation.

\footnotetext{
*e-mail: charles.hudin@cea.fr

†e-mail:jose.lozada@cea.fr

${ }^{\ddagger}$ e-mail:vincent.hayward@isir.upmc.fr
}

In the reference [8], we analysed from first principles the conditions under which computational time reversal could be applied to tactile stimulation. We also exemplified this technique in the case of a stretched BOPET membrane (biaxially-oriented polyethylene terephthalate). In this application, using a membrane as a propagation medium is quite demonstrative but it is not practical, owing to the drift in wave propagation velocity in the face of small mechanical and thermal instabilities. In the present article, we describe the application of time reversal to a cavity made of an ordinary sheet of glass, consumer-electronics-grade piezoelectric actuators, and simplified signal processing. Collectively, these improvements yield a system demonstrating much greater practically. We then show by a simple detection and discrimination study that this simplified equipment can elicit an easily detectable tactile sensation localized within a $1 \mathrm{~cm}^{2}$ region.

\section{Operation Principle and Realization}

\subsection{Brief Recall of the Time Reversal of a Wave Field}

For the sake of exposition, consider first a reverberant cavity with no attenuation. The wave propagation equation, $\partial^{2} u / \partial t^{2}=c^{2} \nabla^{2} u$, with $u$ denoting material displacement, and $t$ denoting time, involves even-order time derivatives only. The solutions, $\phi(x, t)$, of a given propagation problem, including its initial and boundary conditions, are thus symmetrical, that is, $\phi(r, t)=\phi(r,-t)$. Since the time-reversed, or time-symmetric field, $\phi(r,-t)$ is a solution of the same problem, it propagates as time grows until reaching the initial condition at time $t=0$. If this initial condition is an impulse force exerted at some point of the domain, the direct solution $\phi(r, t)$ is a diverging wave-front while the time reversed one would be a converging wave-front. The energy that propagated from a point to the whole volume can be back-propagated to its initial point.

At first sight, the creation of a time-reversed wave-front seems to require an entire direct solution record at every point of the domain. In particular, intuitively, such process would also require a very large collection of transducers able to sample space sufficiently finely. Surprisingly, such is not the case. The Fresnel-Kirchhoff integral theorem states that a wave field inside a volume can be entirely retrieved from the value of the solution and from that of its first derivative on a surface enclosing it. In fact, it is possible to use a finite set of transducers on the boundary of a domain to record the direct field and then to reconstruct an approximation of the same time-reversed field. This insight is behind the fundamental principle of the computational time-reversal of an acoustic field, as initially described by Fink and collaborators [6, 23].

Accounting for reflections of waves at the boundaries of the domain leads to the notion of virtual sources and sinks, just like the reflection of light on a mirror leads to the notion of virtual sources. With properly designed cavities, it is possible to reduce the number of transducers down to a single one to achieve the approximate reconstruction of a field by computational time-reversal [5]. This reduction comes with a tradeoff, however. The number of useful virtual transducers is related to the number of wave reflections on the boundaries the domain, and by consequence to the duration, $T$, 
of the recorded signal of the direct field.

Generally speaking, the quality of the focusing process can be quantified with reference to an ideal delta function. An important measure of quality is the contrast ratio, $C$, the ratio of the amplitude reached at the focused point over the RMS value of displacement elsewhere. The contrast ratio in general increases with the number of transducers, $N$, either real or virtual. It is also a function of the recording time, $T$, but up to a limit. Beyond a given time, $T_{c}$, characteristic of a particular cavity, new information brought about by additional virtual transducers becomes redundant and the contrast ratio tappers off.

The characteristic time is equal to the inverse of the average difference of the frequencies of consecutive modes, that is, it is related to the modal density of the cavity $T_{c}=1 / \Delta f$. A further limit to contrast enhancement when $T$ becomes greater than the attenuation characteristic time, $\tau$, beyond which no new information is added. The amount of information contained per unit duration of signal is also related to its bandwidth, $B$. Previously cited references show that in a $2 \mathrm{D}$ cavity, the contrast ratio obeys

$$
C \simeq \sqrt{B N T}
$$

as long as $N T \ll T_{c}$ and $T \ll \tau$, while the amplitude at focused point $U_{\text {peak }}$ obeys,

$$
U_{\text {peak }} \propto B N T,
$$

as long as $T \ll \tau$.

The reader is referred to [8] for more details regarding the various engineering tradeoffs needed to achieve computational timereversal for use in tactile stimulation.

\section{Physical Realization}

\subsection{Mechanical design}

We used a rectangular glass sheet of dimensions $63 \times 102 \mathrm{~mm}^{2}$ and thickness $0.2 \mathrm{~mm}$. Its edges sides were bonded to a rigid supporting frame. The optimal thickness of piezoelectric transducers, when used to actuate a glass plate is $1 / 4$ of the plate thickness [9]. The diameter of the transducers limit the shortest usable wavelength. The selected transducers were eight piezoelectric diaphragm (Murata 7BB-12-9) with a diameter of $12 \mathrm{~mm}$ and a thickness of $0.120 \mathrm{~mm}$. Their bottom electrode layers were electrically grounded and bonded with epoxy resin to the glass surface at a distance $d \simeq 5 \mathrm{~mm}$ of sides and with a distribution depicted in fig. 1. An uneven distribution of transducers aims at avoiding symmetries that would give correlated waveforms. The first resonance frequency of the device was $f_{0}=170 \mathrm{~Hz}$. The attenuation time, $\tau=3 \mathrm{~ms}$, was found by fitting a decreasing exponential on the envelope of an impulse response. The characteristic time, $T_{c}=10 \mathrm{~ms}$, was calculated in the same way as in [8]. The sampling frequency was $F_{S}=50 \mathrm{kHz}$, giving a bandwidth $B=25 \mathrm{kHz}$.

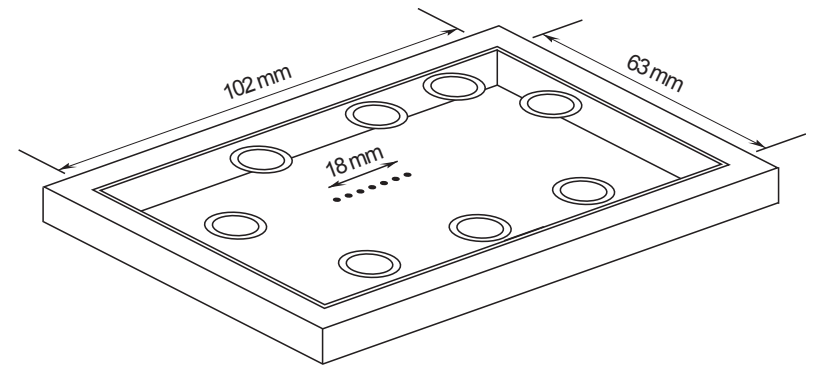

Figure 1: Geometrical parameters of the experimental setup. The double circles represent the $12 \mathrm{~mm}$ actuators. The dots indicate the re-focusing positions used in the perceptual validation described in Section 4.

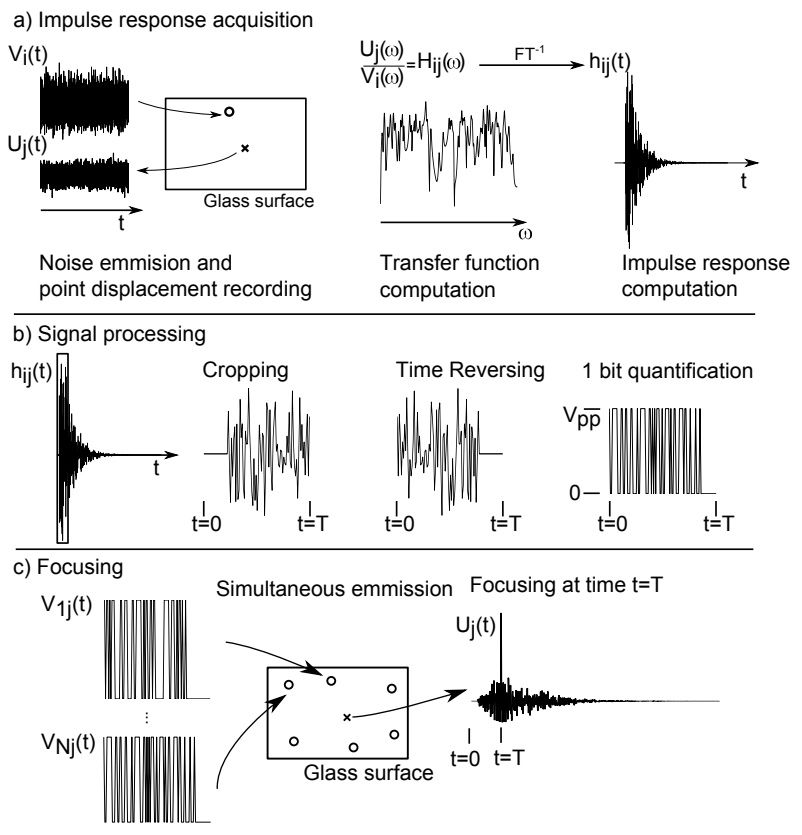

Figure 2: Procedure followed to focus waves at a point j. Impulse responses are computed and processed individually for each actuators. During the focusing step, corresponding voltages are applied simultaneously to all actuators.

\subsection{Signal acquisition and processing}

The first step of a time reversal process is to record the direct propagation field. Since the aim is to create a localised tactile feedback, the appropriate direct field is the response to a point displacement.

The propagation equation is linear, therefore any shape can be created on the surface by summing the weighted contributions of responses at individual points. It is therefore sufficient to record the impulse responses, $h_{i j}(t)$, between each pair of points, $j$, and transducers, $i$. The reciprocity principle can be advantageously applied to ease the recording step. This principle states that the electric potential obtained across the electrodes of any transducer, $i$, resulting from the displacement of any point, $j$, is identical to the displacement measured at point $j$ once an impulse of potential is applied to transducer $i$. Therefore, there is no need to stimulate the surface with a hard-to-achieve displacement impulses in order to record the responses at the transducer locations, but rather to apply voltage impulses to the actuators, which is easy, and to record the displacement at point $j$. Since piezoelectric transducers play the role of actuators both for recording and for reproduction, the driving electronics is greatly simplified, and the need to create calibrated and repeatable mechanical impulses at locations of the surface is avoided.

However, since the voltage impulses ought to be brief to increase signal bandwidth, the energy injected in the plate is low, causing a noisy measurement. To avoid this issue, the input voltage, $V_{i}(t)$, was replaced by a pulse of white noise. By recording the displacement, $U_{j}(t)$, of the surface, the impulse response $h_{i j}(t)$ can be computed from its definition,

$$
h_{i j}(t)=F T^{-1}\left[H_{i j}(\omega)\right]=F T^{-1}\left[\frac{\widehat{U}_{j}(\omega)}{\widehat{V}_{i}(\omega)}\right],
$$

where $H_{i j}(\omega), \widehat{U}_{j}(\omega)$ and $\widehat{V}_{i}(\omega)$ denotes the Fourier transform of $h_{i j}(t), U_{j}(t)$ and $V_{i}(t)$ respectively and $F T^{-1}$ is the inverse Fourier transform operation. This operation is repeated for each transducer and each point of the surface. The time reversal operation then 


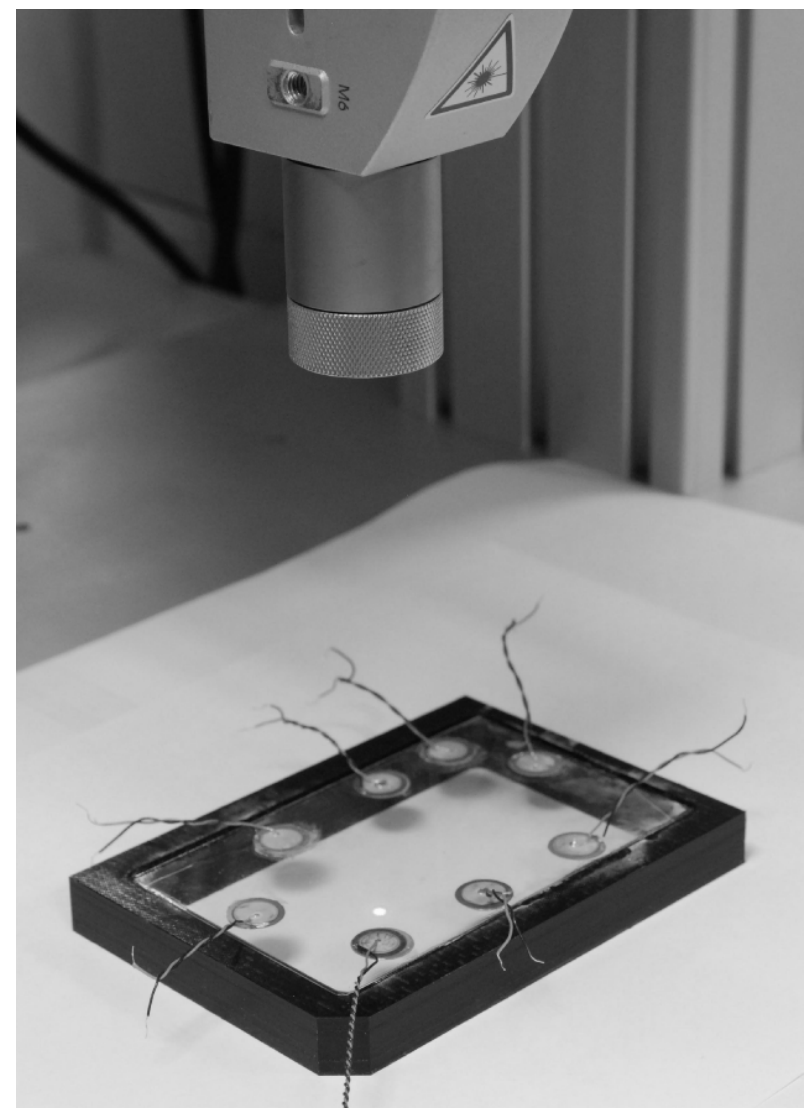

Figure 3: Setup picture. The laser vibrometer is mounted on a threeaxis motorised table to measure the surface displacement of the device at a given position.

consists on sending to each actuator, $i$, the time reversed signal $h_{i j}(T-t)$ corresponding to the point, $j$.

Lastly, it has been shown that the signal phase is the most important parameter to achieve good focusing [15], to the point that amplitude information can be compressed to just the sign of the signal, that is, to just one level of quantification. Thus, single-bit driving electronics suffice and since the result is not sensitive to dc component, the need for a bipolar power supply can also be avoided. As an added bonus, the square driving signal injects more energy into the plate than an amplitude modulated signal, leading to a higher peak amplitude for the same contrast ratio. The voltage applied to actuator $i$ in order to re-focus at point $j$ is thus given by

$$
V_{i j}(t)=\frac{1}{2} V_{\mathrm{pp}}\left(1+\operatorname{sign}\left(h_{i j}(T-t)\right)\right),
$$

where $V_{\mathrm{pp}}$ is the peak-to-peak voltage.

To achieve the focusing at a point $\mathrm{j}$, voltages $V_{i j}(t)$ are applied simultaneously to each actuators i. Figure 2 summarize this acquisition and focusing procedure.

\subsection{Physical Validation}

The performance in terms of peak spatial spread and amplitude are reported in this section. Two cases were considered according to whether or not a finger touched the surface.

\subsubsection{Unloaded Performance}

In a first test, the duration of the time reversal window was set to $T=2 \mathrm{~ms}$ and the peak-to-peak voltage to $V_{\mathrm{pp}}=24 \mathrm{~V}$. A laser vibrometer (Polytec OFV 534) mounted on a three-axis motorised table was moved across the surface, by steps of $2 \mathrm{~mm}$. Figure 3 illustrates the vibrometer pointing a laser beam at the surface.

The same focusing operation was performed at each position while the actuator driving voltage and the point displacement, integrated from velocity, were recorded at a sampling rate of $100 \mathrm{kHz}$. The driving voltage was the same for all focusing operations. Its onset served as a reference from which the vibrometer signal could be synchronised. Owing to the good repeatability of the process, a reconstruction of the experimental focusing could be performed. Figure 4 shows the reconstructed displacement of the surface at time $T=2 \mathrm{~ms}$ at which the focusing occurs. The focus point is clearly visible with an amplitude of $12 \mu \mathrm{m}$ and a diameter about $10 \mathrm{~mm}$.

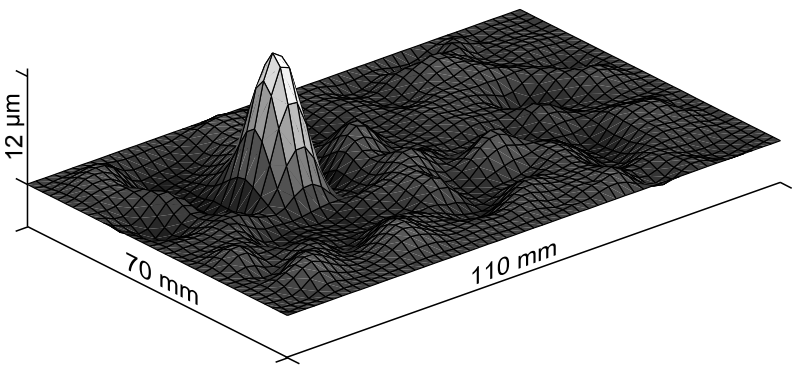

Figure 4: Experimental out of plane displacement at focus time for a driving voltage amplitude $V_{\mathrm{pp}}=24 \mathrm{~V}$.

The measurements, see fig. 5, show the proportionality of the amplitude, $U_{\text {peak }}$, reached at the focus point to the driving voltage, $V_{\mathrm{pp}}$. In this configuration and for $T=2 \mathrm{~ms}$, the gain was $G=U_{\text {peak }} / V_{\text {pp }} \simeq 0.5 \mu \mathrm{m} / \mathrm{V}$. At the maximum operating voltage, $V_{\mathrm{pp}}^{\max }=54 \mathrm{~V}$, which is close to the transducers admissible voltage, the amplitude at the focus point was $U_{\text {peak }}^{\max }=28 \mu \mathrm{m}$. The contrast ratio, however, was not affected by the driving voltage, since peak and background noise amplitude were affected equally. For this setup and for $T=2 \mathrm{~ms}$, the measured contrast ratio was $C \simeq 15$ for only eight actuators.

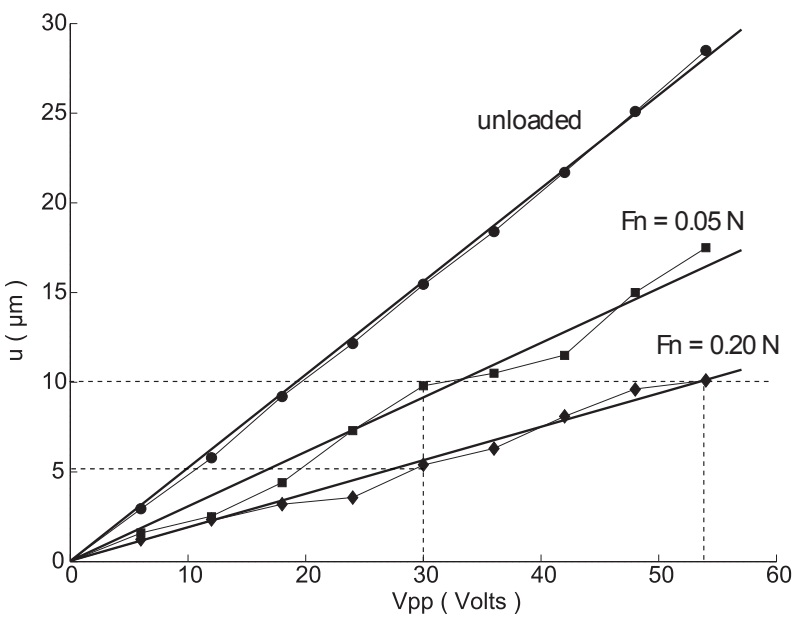

Figure 5: Displacement measurements as a function of the driving voltage amplitude $V_{\mathrm{pp}}$. Amplitude shown in the absence of finger contact (circles), pressing at $0.05 \mathrm{~N}$ (squares) and at $0.20 \mathrm{~N}$ (diamonds). For $V_{p p}=30 \mathrm{~V}$, a displacement of 5 to $10 \mu \mathrm{m}$ is obtained according to the finger contact force. Achieving a diplacement of $10 \mu \mathrm{m}$ requires a driving voltage amplitude between 30 and $55 \mathrm{~V}$. 


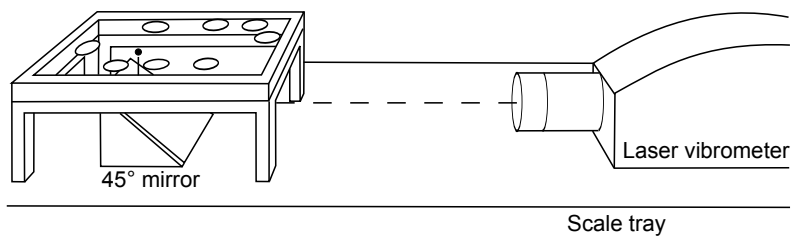

Figure 6: Set up for the measurement of finger normal force and plate displacement under the finger. The vibrometer laser beam is deflected by the mirror and reflected on the bottom side of the glass plate.

\subsubsection{Finger Loaded Performance}

Touching the active surface muffled some of the vibration and actual displacement of the finger pulp was thus smaller than the unloaded response. Therefore, we proceeded in measuring the fingerloaded response.

The attenuation factor was expected to be dependent on finger impedance and contact area [8]. The plate displacement was measured by pointing the vibrometer beam on the bottom side of the plate, while the finger pressed the plate at the same position on the opposite side. The entire assembly was set on a precision scale, see fig. 6.

Figure 7 shows the displacement measured with the loaded and unloaded plate at the focused point and away from it for a loading force $F=200 \mathrm{mN}$. From this figure we can see that the displacement is reduced by a factor three when the plate is loaded by a finger and that this reduction factor affects the peak amplitude as well as the background noise in similar proportions. The contrast ratio is therefore not affected by the presence of a finger.
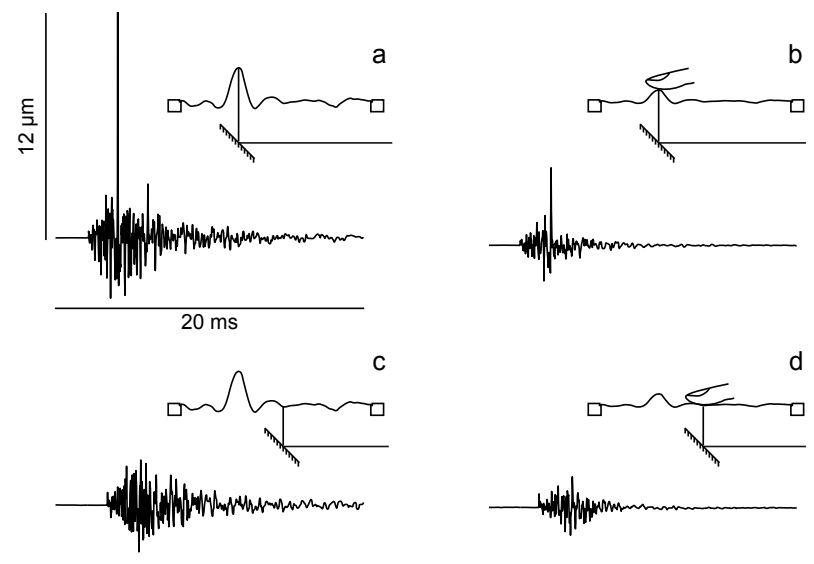

Figure 7: Displacement obtained for $V_{p p}=24 \mathrm{~V}$ and $T=2 \mathrm{~ms}$ at focused point in the absence(a)/presence(b) of a finger and at a point away from focusing in the absence(c)/presence(d) of a finger pressing at $F=0.2 \mathrm{~N}$.

This measurement was repeated for various driving voltage amplitudes and for load forces of $F n=0.05 \mathrm{~N}$ and $F n=0.20 \mathrm{~N}$.

These values were considered to be in the reasonable range of tactile exploration. The results are seen on fig. 5. This figure shows that, loaded or not, the plate displacement is proportional to the driving voltage amplitude. Thus, there is also proportionality between the displacement reached when exerting a constant normal force and the displacement in the absence of finger contact. This proportionality factor is however dependent on the force applied and is approximately $U_{\text {loaded }} / U_{\text {unloaded }}=R=0.3$ for a $0.2 \mathrm{~N}$ load and $R=0.5$ for $0.05 \mathrm{~N}$ load. This means that the presence of a finger induces both an attenuation of the amplitude and a dependence of this amplitude with respect to the load force.

\section{Perceptual validation}

Two testing procedures were carried to demonstrate the ability of the device to produce noticeable and localised tactile feedback, a detection task to determine whether there was a threshold at which stimulation could be felt and a discrimination task to determine at which distance two nearby stimulation regions could be separated.

\subsection{Intensity Threshold}

A wide variety of parameters could influence stimulus detection. Such parameters include its duration, the repetition frequency, the temporal modulation and so on. We investigated the simplest case, namely, the effect of amplitude since amplitude is the natural control parameter of the device. The detectability of a stimulus can be quantified by determining the threshold value above which observers can detect the stimulus more than 50 percent of its occurrences. We used the method of constant stimuli in an attempt to determine the psychometric function between the probability of stimulus detection and the corresponding driving voltage amplitude $V_{\mathrm{pp}}$.

\subsubsection{Stimuli, Participants, and Procedure}

The stimuli were produced by focusing the waves at position known to the observers at a rate of $150 \mathrm{~Hz}$ during five seconds. The time window of time reversal was $T=2 \mathrm{~ms}$. The amplitude was changed in each trial by selecting it randomly among six uniformly distributed values ranging from $6 \mathrm{~V}$ to $51 \mathrm{~V}$. Seven volunteers, three female and four male, aged 22 to 30 participated in the study. One hundred of these stimuli were presented to each participant and their responses, stimulus/no-stimulus, were recorded. They received no directions as to how to touch the surface and they were allowed to move their finger in the vicinity of the point. They wore isolating headphones playing white noise during the whole duration of the task .

\subsubsection{Results}

The probability of detection of a stimulus was calculated for each participant and each driving voltage amplitude. This probability is, at a given stimuli amplitude, the ratio of the number of detected stimuli over the number of presented stimuli. The dots and the vertical bars on fig. 8 show the average and standard deviation of detection probability over all participants. The continuous line shows the fitted psychometric function $f(x)=\frac{1}{2}[1+\operatorname{erf}((x-t) s / \sqrt{\pi})]$ where $s$ is the function slope at threshold value $t$. As expected, the detection probability increased with driving voltage amplitude. It reached 0.85 , indicating an high rate of detection of the stimuli. The detection threshold can be estimated to be at $V_{\mathrm{pp}}^{\mathrm{th}}=32 \mathrm{~V}$.

\subsubsection{Discussion}

The actual displacement was not measured during this experiment. Figure 7, however, allows us to estimate the displacement to be between 5 and $10 \mu \mathrm{m}$.

This detection threshold might seem quite high when compared to literature values for detection threshold of vibrotactile stimuli [20]. This is likely to come from the very short duration of the impulse. Indeed, the maximum frequency used to build the interference is $f_{\max }=25 \mathrm{kHz}$ which implies that the impulse duration is $T_{i}=1 / 25000=40 \mu \mathrm{s}$. Because of this frequency range and the impulsive nature of the stimuli, comparison with detection threshold of sinusoidal vibrotactile stimuli does not seem appropriate.

Such frequencies indeed fall well above vibrotactile sensibility range and should at first sight prevent the impulse from being felt. One hypothesis, that requires further investigations, would be that the high acceleration resulting from the briefness of the impulse, 


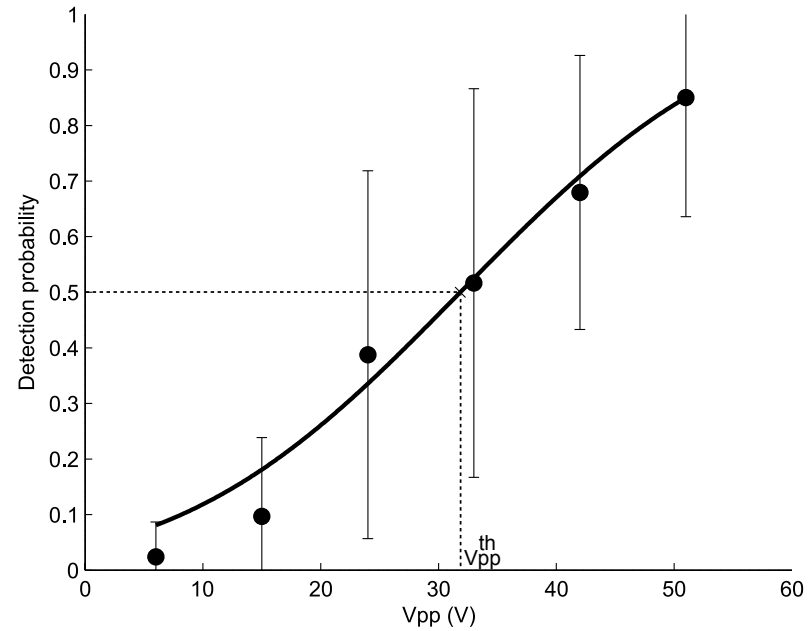

Figure 8: Probability of detection of a focused point for various driving voltage amplitudes

induces some non linear effects, such as skin/plate separation, leading to a finger skin deformation much longer than the actual stimuli duration.

The large standard deviation seen on these results denotes a significant spread in sensitivity among the participants. This effect can be explained by the device's sensitivity to forces exerted by the users during exploration. Figure 5 shows that a driving voltage between 30 and 55 Volts is needed to achieve a $10 \mu \mathrm{m}$ with a finger-loaded plate and that this figure can vary according to the force applied.

Assuming that this dependence comes from the change in finger impedance with changing load force, the simplified model of interaction between the plate vibrations and the finger proposed in [8] can be invoked. According to this model, the ratio, $R$, of loaded by unloaded displacements is $R=2 Z_{S} /\left(Z_{F}+2 Z_{S}\right)$, where $Z_{S}$ and $Z_{F}$ are the surface and finger impedance. The rate of change of $R$ with respect to changes in finger impedance is then $d R / d Z_{F}=$ $-2 Z_{S} /\left(2 Z_{S}+Z_{F}\right)^{2}$. Consequently, increasing the impedance of the surface, $Z_{S}$, would increase $R$ and also reduce its sensitivity with regard to the finger impedance. With an larger surface impedance, the finger displacement would be kept more constant with respect to possible perturbations. The price to pay would be a higher power consumption.

The fact that the detection probability of 1.0 was not achieved indicates that the amplitude provided by the device could be increased to facilitate the detection either by increasing unloaded plate peak displacement or the transmission ratio $R$.

\subsection{Separable Distance}

The focal spot produced by a time reversal process has a finite size, given by the diffraction limit which is therefore related to the signal bandwidth, leading to a finite display resolution. Here we aimed at measuring the minimum distance between stimuli so that they could be separated.

\subsubsection{Stimuli, Participants, and Procedure}

Seven points, depicted in fig. 1, were uniformly distributed along a line with a $3 \mathrm{~mm}$ spacing. Point one was systematically focused alternatively, either with itself or with one elected among the six other possibilities. The driving voltage was $V_{p p}=51 \mathrm{~V}$. Again, a constant stimulus method was employed, giving a probability of 0.5 for the perfect detection of two distinct points. Time reversal could focus simultaneously two or more points by summing the signals but the same total energy would have to be divided by the number of points. On the other hand, the decay time constant of the cavity of $\tau=3 \mathrm{~ms}$ made it possible to repeat the focusing process at a rate up to 300 $\mathrm{Hz}$ without increasing the background noise level. The two points could thus be focused alternatively at a rate of $150 \mathrm{~Hz}$ each, with a time reversal window of $T=2 \mathrm{~ms}$. The same seven subjects participated. One hundred pairs of points were presented for five seconds. The locations of extreme points were known to the participants and they were free to explore the region in-between before answering whether they felt two distinct stimuli. Likewise, they wore isolating headphone playing white noise during the whole duration of the task.

\subsubsection{Results}

The probability to distinguish two stimuli was calculated for each participants. Figure 9 shows the average and standard deviation over participants, as a function of distance between focused points. The discrimination threshold was found to $d^{\text {th }}=10 \mathrm{~mm}$.

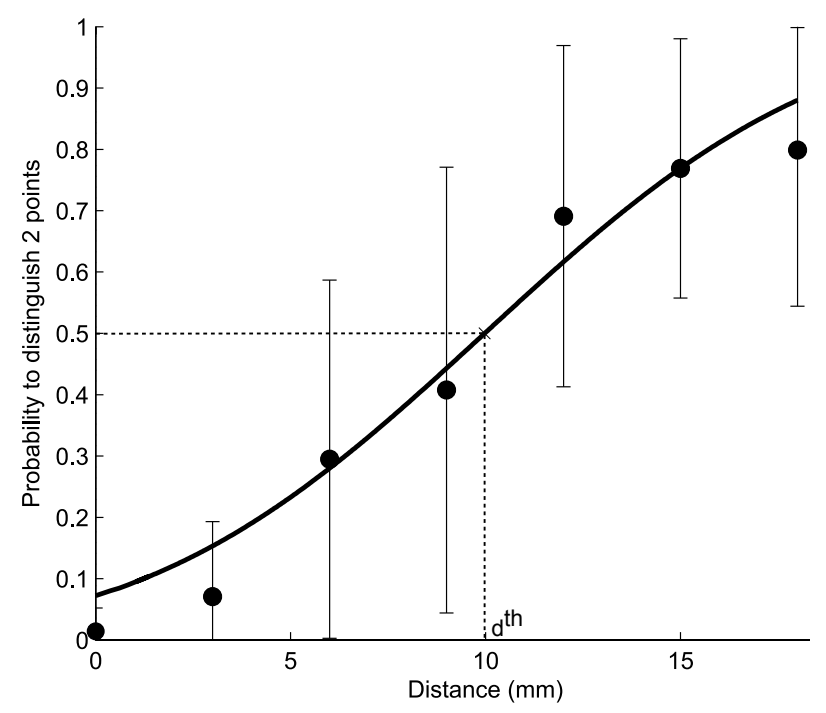

Figure 9: Probability of discrimination of two focused point as a function of their relative distance

\subsubsection{Discussion}

As could be expected, the discrimination probability increased with the distance between points. The existence of a discrimination threshold validates the localization capabilities of the device. The notion that the peak amplitude was actually differentiated from the background noise was reinforced by the finding that distance threshold value of $10 \mathrm{~mm}$ which coincides with the spread of a focused point observed on fig. 4 . The probability to discriminate the location of two points does not reach one even for the most distant points. This is likely due to the detection probability of a single stimuli for $V_{p p}=51 \mathrm{~V}$ found in the first study to be bellow unity. Thus the probability of not detecting one of points is not zero, no matter how distant they are from each other.

\section{Conclusion}

A functional tactile display based on computational time-reversal and using a glass surface and consumer-grade actuators was demonstrated. It operated from simplified data acquisition and processing. This simplified procedure appealed to the reciprocity principle and to the ability of piezoelectric transducers to act as sensors or as actuators, avoiding the need for mechanical calibration of the 
impulse responses. A second innovation was the use of singlelevel electronics to drive the actuators, achieving enormous cost and complexity reduction. Physical measurement combined with a user study showed the produced stimuli were clearly detectable and confined to a region of about $1 \mathrm{~cm}^{2}$ area, thus validating the effectiveness of the time reversal approach to display localised tactile informations. Further research will aim at transposing these results to the ultrasound frequency range to avoid sound production. The influence of finger on the focusing quality will be also further investigated.

\section{REFERENCES}

[1] O. Bau, I. Poupyrev, A. Israr, and C. Harrison. TeslaTouch: electrovibration for touch surfaces. In Proceedings of the 23nd annual ACM symposium on User interface software and technology, pages 283-292, 2010.

[2] M. Biet, F. Giraud, and B. Lemaire-Semail. Squeeze film effect for the design of an ultrasonic tactile plate. IEEE Transactions on $\mathrm{Ul}$ trasonics, Ferroelectrics and Frequency Control, 54(12):2678-2688, 2007.

[3] J. L. Burke, M. S. Prewett, A. A. Gray, L. Yang, F. R. B. Stilson, M. D. Coovert, L. R. Elliot, and E. Redden. Comparing the effects of visual-auditory and visual-tactile feedback on user performance: a meta-analysis. In Proceedings of ICMI 2006, pages 108-117, 2006.

[4] E. C. Chubb, J. E. Colgate, and M. A. Peshkin. Shiverpad: A glass haptic surface that produces shear force on a bare finger. IEEE Transactions on Haptics, 3(3):189-198, 2010.

[5] C. Draeger, J. Aime, and M. Fink. One-channel time-reversal in chaotic cavities: Experimental results. The Journal of the Acoustical Society of America, 105:618, 1999.

[6] M. Fink. Time reversal of ultrasonic fields. i. basic principles. Ultrasonics, Ferroelectrics and Frequency Control, IEEE Transactions on, 39(5), 1992.

[7] F. Giraud, M. Amberg, B. Lemaire-Semail, and G. Casiez. Design of a transparent tactile stimulator. In Proceedings of the Symposium on Haptic Interfaces For Virtual Environment And Teleoperator Systems, pages 485-489, 2012.

[8] C. Hudin, J. Lozada, M. Wiertlewski, and V. Hayward. Tradeoffs in the application of time-reversed acoustics to tactile stimulation. Haptics: Perception, Devices, Mobility, and Communication, pages 218226, 2012.

[9] J. Jones and S. Kim. Optimal design of piezo-actuators for active noise and vibration control. In AIAA, Space Programs and Technologies Conference, volume 1, 1990.

[10] L. A. Jones and N. B. Sarter. Tactile displays: Guidance for their design and application. Human Factors: The Journal of the Human Factors and Ergonomics Society, 50(1):90-111, Feb. 2008.

[11] S. Y. Kim, J. O. Kim, and K. Kim. Traveling vibrotactile wave-a new vibrotactile rendering method for mobile devices. Consumer Electronics, IEEE Transactions on, 55(3):1032-1038, 2009.

[12] V. Lévesque, L. Oram, K. MacLean, A. Cockburn, N. D. Marchuk, D. Johnson, J. E. Colgate, and M. A. Peshkin. Enhancing physicality in touch interaction with programmable friction. In Proceedings of ACM Conference on Human Factors in Computing Systems (CHI'11), pages 2481-2490, 2012.

[13] K. E. MacLean. Putting haptics into the ambience. IEEE Transactions on Haptics, 2(3):123-135, 2009.

[14] E. Mallinckrodt, A. L. Hughes, and W. S. Jr. Perception by the skin of electrically induced vibrations. Science, 277-278, 1953.

[15] G. Montaldo, P. Roux, A. Derode, C. Negreira, and M. Fink. Generation of very high pressure pulses with 1-bit time reversal in a solid waveguide. The Journal of the Acoustical Society of America, 110(6):2849, 2001.

[16] A. Oulasvirta, S. Tamminen, V. Roto, and J. Kuorelahti. Interaction in 4-second bursts: the fragmented nature of attentional resources in mobile HCI. In Proceedings of CHI 2005, the International Conference on Human Factors in Computing Systems, pages 919-928, 2005.

[17] J. Pasquero and V. Hayward. Tactile feedback can assist vision in mobile interaction. In Proceedings of CHI 2011, the International
Conference on Human Factors in Computing Systems, pages 32773280, 2011.

[18] R. M. Strong and D. E. Troxel. An electrotactile display. ManMachine Systems, IEEE Transactions on, 11(1):72-79, 1970.

[19] H. Tang and D. Beebe. A microfabricated electrostatic haptic display for persons with visual impairments. IEEE Transactions on rehabilitation engineering, 6(3):241-248, 1998.

[20] R. Verrillo, A. Fraioli, and R. Smith. Sensation magnitude of vibrotactile stimuli. Attention, Perception, \& Psychophysics, 6(6):366-372, 1969.

[21] T. Watanabe and S. Fukui. A method for controlling tactile sensation of surface roughness using ultrasonic vibration. In Proceedings of the IEEE International Conference on Robotics and Automation, pages 1134-1139, 1995.

[22] M. Wiertlewski, J. Lozada, E. Pissaloux, and V. Hayward. Causality inversion in the reproduction of roughness. Haptics: Generating and Perceiving Tangible Sensations, pages 17-24, 2010.

[23] F. Wu, J. Thomas, and M. Fink. Time reversal of ultrasonic fields. II. experimental results. Ultrasonics, Ferroelectrics and Frequency Control, IEEE Transactions on, 39(5):567-578, 1992. 